\title{
FACTORES ASOCIADOS AL ASEGURAMIENTO PÚBLICO DE GESTANTES EN LA DIRECCIÓN REGIONAL DE SALUD TACNA-2014
}

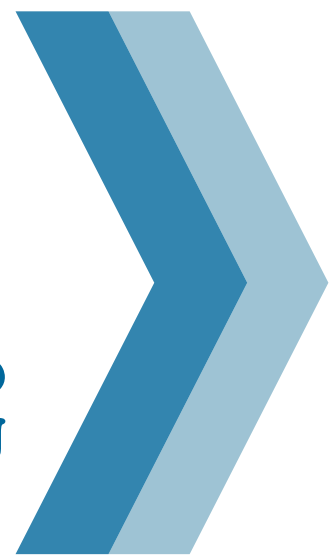

\author{
FACTORS ASSOCIATED WITH \\ PUBLIC HEALTH INSSURANCE \\ OF PREGNANT WOMEN IN THE \\ R E G I O N A L H E A L T H \\ DIRECTORATE OF TACNA-2014
}

\section{Patricia Verónica Montalvo Chávez}

1. Doctora en epidemiologia. Coordinadora Regional de la Escuela de Posgrado de la Universidad César Vallejo, sede Tacna.

\section{RESUMEN}

OBJETIVO: Determinar los factores asociados al aseguramiento público subsidiado de las gestantes en la DIRESA Tacna durante el año 2014. MATERIALES Y MÉTODOS: Estudio analítico, comparativo, no experimental, prospectivo. Se encuestó a 346 gestantes de la DIRESA Tacna, seleccionadas de una población de 5723 gestantes, utilizando la ecuación de Cochran. Se excluyó a las gestantes que no deseaban participar del estudio. Se creó un instrumento de recolección de datos, el cual fue validado mediante juicio de expertos; seguidamente, este fue aplicado como encuestas a las gestantes. El procesamiento de la información y análisis estadístico se realizó usando el programa SPSS versión 24. RESULTADOS: Solo el $35.26 \%$ de gestantes presentó aseguramiento público. Según la condición de aseguramiento, el $20.52 \%$ del grupo etario entre 20 a 34 años contaba con seguro de salud, versus el $32.95 \%$ de gestantes no aseguradas. El $26.88 \%$ de convivientes contaba con seguro de salud. Además, las gestantes solteras contaban con $2.2 \%$ mayor riesgo de no asegurarse respecto a las casadas $(p<0.05)$. Sobre la oportunidad en el aseguramiento, la brecha se acortaba a mayor edad gestacional, existiendo interés solo para el parto. El $87.28 \%$ de gestantes cursaban la gestación sin un factor de riesgo. El $58.95 \%$ del personal de salud responsable del aseguramiento desconoce el proceso y atendió al $88 \%$ de gestantes sin acceso al seguro gratuito $(p<0.05)$. El $80.06 \%$ de gestantes solicitaron el seguro por primera vez. CONCLUSIÓN: La cobertura de afiliación de gestantes al aseguramiento público fue baja. No existe relación entre esta situación con el grado de instrucción, factores de riesgo y factores económicos de las gestantes. EI aseguramiento no es oportuno y se da principalmente en el último trimestre del embarazo. Finalmente, el personal de salud responsable del aseguramiento desconocía los procesos del mismo.

Palabras clave: Aseguramiento en gestantes, factores de riesgo en gestantes.

\section{ABSTRACT}

OBJECTIVE: To determine the factors associated with subsidized public insurance for pregnant women in the DIRESA Tacna during 2014. MATERIALS AND METHODS: Analytical, comparative, non-experimental, prospective study. We interviewed 346 pregnant women from DIRESA Tacna, selected from a population of 5723 pregnant women, using the Cochran equation. Pregnant women who did not want to participate in the study were excluded. A data collection instrument was created, and it was validated by expert judgment. Then this instrument was applied as surveys to pregnant women. The information processing and statistical analysis was performed using the program SPSS version 24. RESULTS: Only $35.26 \%$ of pregnant women presented public insurance. According to the insurance condition, $20.52 \%$ of the group aged 20 to 34 years old had insurance, versus the $32.95 \%$ of uninsured pregnant women. The $26.88 \%$ of cohabitants had health insurance. In addition, unmarried pregnant women had a 2.2 higher risk of not being insured compared to married women $(p<0.05)$. Regarding the opportunity in the insurance, the gap was shortened at a higher gestational age, existing interest only for childbirth. The $87.28 \%$ of pregnant women went through pregnancy without a risk factor. The $58.95 \%$ of the health personnel responsible for the insurance ignored the process and they treated $88 \%$ of pregnant women without access to free insurance $(p<0.05)$. The $80.06 \%$ of pregnant women applied for the insurance for the first time. CONCLUSION: The coverage of pregnant women's affiliation to public insurance was low. There was no relation between this situation with the degree of education, risk factors and economic factors of the pregnant women. The insurance was not timely and occurred mainly in the last trimester of pregnancy. Finally, the staff responsible for the insurance ignored the processes of the same.

Keywords: Insurance in pregnant women, risk factors in pregnant women. 


\section{INTRODUCCIÓN}

En el Perú se viene trabajando para mejorar la situación sanitaria del país, prioritariamente la disminución de la mortalidad materna como una meta clave de desarrollo. Con tal propósito, el Ministerio de Salud, como órgano rector, ha implementado el "Plan Estratégico para Reducir la Mortalidad"(1), con el conocimiento de que la hemorragia es la primera causa de muerte materna; sin embargo se conoce también que esta situación es prevenible. Por tanto, debe garantizarse el acceso a los servicios de salud a las gestantes. Del Carpio ${ }^{(2)}$ afirma que el incremento del acceso a los servicios de salud se debe al aseguramiento en salud, la cual tiene efecto directo en los indicadores de morbi- mortalidad de las gestantes. Según Misas ${ }^{(3)}$, se han realizado una serie de reformas a los sistemas de salud con el propósito de mejorar las condiciones de salubridad y potenciar los planes de salud que cubran las principales enfermedades y dolencias de las personas, dado un perfil epidemiológico. Parodi ${ }^{(4)}$ en su estudio "Evaluando los Efectos del Seguro Integral de Salud (SIS) sobre la Equidad en la Salud Materna", en el contexto de barreras no económicas al acceso a los servicios, afirma que una considerable proporción ( $2 / 3$ del total) se encuentra afiliadas al SIS, que va del $87.5 \%$ en el quintil 1 al $25.0 \%$ en el quintil 5 .

En el ámbito internacional se encuentran estudios previos sobre seguros como es el caso de México que cuenta con un seguro popular ${ }^{(5)}$. Ferreira, en Uruguay, investiga sobre oportunidades en la captación de gestantes para las atenciones en salud en su estudio "Características del Control Prenatal en las Embarazadas Adolescentes" ${ }^{\text {(6) }}$. Becerril ${ }^{(7)}$ indica que la mitad de la población carecía de cobertura sanitaria en Brasil en el año 1988. Posteriormente, transcurridas dos décadas desde que se estableció su Sistema Único de Salud, más del 75\% dependía exclusivamente de él para su atención médica. En Bolivia, según un informe del Ministerio de Justicia en el año $2002^{(8)}$, las principales políticas de salud de los últimos años se concentraron en la atención a mujeres y niños a través de sistemas de aseguramiento público con el propósito de reducir las elevadas tasas de mortalidad, morbilidad y prevención de riesgos de estos grupos.

En el Perú encontramos estudios como la "Evaluación del Efecto del Seguro Integral de Salud en los Indicadores de Salud Pública y Gasto de Bolsillo Periodo 2002-2009"(9) y el desarrollado por la Defensoría del Pueblo "Informe de Salud sobre la Equidad de Atención de Salud para los Más Pobres" ${ }^{\prime(10)}$.
Adicionalmente, diversos autores abordaron esta temática, tales como Santos ${ }^{(11)}$, quien estudio factores que limitaban la cobertura de gestantes controladas afiliadas al seguro integral de salud; Munares ${ }^{(12)}$ estudió la calidad del control prenatal; Navarro ${ }^{(13)}$ investigó factores de riesgo para el inicio tardío en el control prenatal; ya $\mathrm{Chaw}^{(14)}$, en su artículo de aseguramiento universal, encontró que solo el $35 \%$ tenía acceso a un seguro de salud.

A nivel regional, Montalvo \& Galdos ${ }^{(15)}$ estudiaron el efecto del aseguramiento público en los indicadores materno infantiles de la Región Tacna, lo cual resultó ser positivo. En Trujillo, Ávalos ${ }^{(16)}$ encontró que el $65.6 \%$ de las gestantes contaban con un seguro público como es el Seguro Integral de Salud.

El objetivo del presente estudio fue determinar los factores asociados al aseguramiento público subsidiado de las gestantes en la Dirección Regional de Salud Tacna durante el año 2014.

\section{MATERIALES Y MÉTODOS}

El presente estudio es de tipo analítico, comparativo, no experimental y prospectivo. Se evaluaron, los conocimientos del personal de salud que realiza los procesos de afiliación y el equipamiento de los establecimientos de salud. La población estuvo constituida por 5723 gestantes de la Región Tacna durante el año 2014 según datos del INEI ${ }^{(16)}$ de las cuales se estudió una muestra de 346 gestantes. La ecuación de Cochran fue utilizada para estimar el tamaño de la muestra. Adicionalmente, se creó un instrumento de recolección de datos, validado mediante juicio de expertos. Este instrumento se aplicó como encuesta a las gestantes. El procesamiento de la información y análisis estadístico se realizó usando el programa SPSS versión 24.

\section{RESULTADOS}

Se encontró que solo el $35.26 \%$ de las gestantes contaba con un seguro de salud, es decir 1 de cada 3 gestantes estaría protegida por un seguro de salud y con prestaciones de salud garantizadas gratuitamente (Tabla 1)

\section{Tabla 1: Cobertura de aseguramiento en gestantes en} la DIRESA Tacna, 2014

\begin{tabular}{lcc} 
Aseguramiento & Número & Porcentaje \\
\hline No asegurada & 224 & 64.74 \\
Asegurada & 122 & 35.26 \\
Total & 346 & 100.00 \\
\multicolumn{2}{c}{$\quad$ Prueba $=\mathrm{X}^{2}$} & $1.1065 \mathrm{p}=0.5751$
\end{tabular}


El grupo predominante de las gestantes se concentró en el último trimestre del embarazo con más de la mitad de las gestantes asistentes a los servicios de salud. Se puso en evidencia que solo el $15.03 \%$ de gestantes se aseguraron en el primer trimestre de gestación, lo cual es una situación óptima (Tabla 2).

\begin{tabular}{|c|c|c|c|c|c|c|}
\hline \multirow{2}{*}{$\begin{array}{c}\text { Edad } \\
\text { Gestacional }\end{array}$} & \multicolumn{2}{|c|}{ Asegurada } & \multicolumn{2}{|c|}{$\begin{array}{c}\text { No } \\
\text { Asegurada }\end{array}$} & \multicolumn{2}{|r|}{ Total } \\
\hline & $\mathbf{N}^{\circ}$ & $\%$ & $\mathbf{N}^{\circ}$ & $\%$ & $\mathbf{N}^{\circ}$ & $\%$ \\
\hline $\begin{array}{c}1 \text { A } 12 \\
\text { SEMANAS }\end{array}$ & 15 & 4.34 & 37 & 10.69 & 52 & 15.03 \\
\hline $\begin{array}{l}13 \text { A } 28 \\
\text { SEMANAS }\end{array}$ & 41 & 11.85 & 71 & 20.52 & 112 & 32.37 \\
\hline $\begin{array}{c}29 \text { A } 43 \\
\text { SEMANAS }\end{array}$ & 66 & 19.08 & 116 & 33.53 & 182 & 52.60 \\
\hline TOTAL & 122 & 35.26 & 224 & 64.74 & 346 & 100.00 \\
\hline
\end{tabular}

El grupo predominante de gestantes tenía estudios de nivel secundario (67.8\%), seguida del nivel superior (19.62\%). Es importante resaltar que en cada grupo de gestantes hubo una proporción de una gestante asegurada frente a dos no aseguradas (Figura 1)

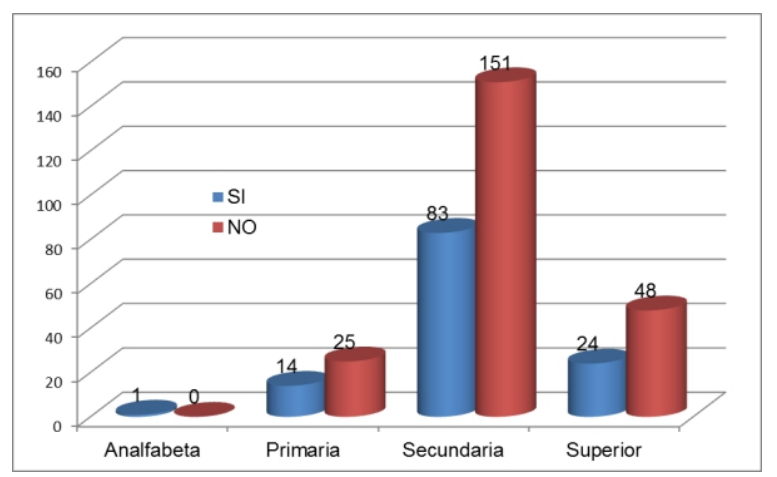

Figura 1. Aseguramiento de gestantes según grado de instrucción en la DIRESA Tacna, 2014.

Con respecto al estado civil, se evidencia que casi las dos terceras partes de las gestantes (69.65\%) fueron convivientes, seguidas del grupo de las solteras con $17.92 \%$ y el grupo de las casadas con el $12.14 \%$. Se observó, además, que las gestantes solteras tuvieron $2.2 \%$ mayor riesgo de no asegurarse respecto a la casada (Figura 2).

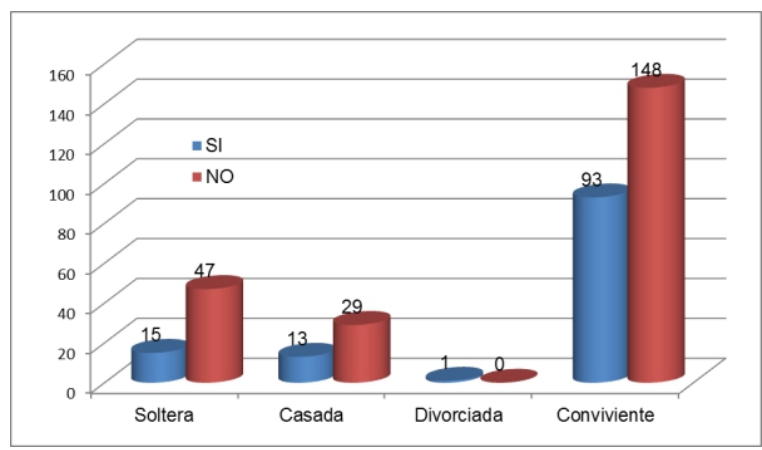

Figura 2. Aseguramiento de gestantes según estado civil en la DIRESA Tacna, 2014.

Por último, encontramos que un poco más de la mitad del personal de salud (58.95\%) responsable del aseguramiento desconocía el proceso. Así, la mayoría (88\%) de gestantes sin acceso al seguro gratuito fueron atendidas por personal que desconocía el proceso de aseguramiento, frente al $95 \%$ de gestantes aseguradas atendidas por personal que sí conocía el proceso (Tabla 3).

\begin{tabular}{|c|c|c|c|}
\hline ASEGURAMIENTO & $\begin{array}{c}\text { NO } \\
\text { ASEGURADA }\end{array}$ & ASEGURADA & Total \\
\hline DESCONOCE & 198 & 26 & 224 \\
\hline CONOCE & 6 & 116 & 122 \\
\hline TOTAL & 204 & 142 & 346 \\
\hline
\end{tabular}

\section{DISCUSIÓN}

Basados en los resultados, se observa que antes del estudio se tenía la percepción de que la cobertura de aseguramiento en gestantes era alta. Esta percepción se acentuó aún más, ya que no se cuenta con valores basales regionales, debido principalmente a la falta de estudios previos referidos a esta temática. Posteriormente, este estudio concluye que la cobertura de aseguramiento en la DIRESA Tacna durante el año 2014 fue baja. Respecto a las limitaciones de este estudio podemos señalar que se encontraron pocos estudios similares al tema que puedan presentarse como antecedentes.

En este estudio, como en el de Vargas ${ }^{(17)}$, se observó que 
la principal barrera de acceso al aseguramiento fue la calidad de información que manejaba y brindaba el personal de salud al paciente, pese al derecho que les asiste de ser informados. Al igual que lo concluido en el estudio del INS de Colombia ${ }^{(18)}$, en este trabajo se encontró que las barreras de acceso económico por falta de un seguro que cubriese las prestaciones en salud, afectaba la oportunidad de atención en la gestante; quienes deberían lograr su aseguramiento en el primer trimestre de gestación.

La similitud entre el presente estudio y el realizado por Saavedra ${ }^{(19)}$ en México, consiste en la aseveración que el aseguramiento público mejora el acceso a los servicios de salud. En el presente estudio se encontró que la cobertura de gestantes aseguradas fue de un $35.26 \%$ en nuestra región Tacna durante el año 2014. Es importante también resaltar que los resultados de este estudio difieren de los obtenidos por Parodi ${ }^{(4)}$, en el cual se determinó que el $66.8 \%$ de las gestantes del Perú durante el año 2005 se encontraban aseguradas en el Seguro Integral de Salud. Porcentaje que constituye dos tercios de la población con cobertura. Sin embargo, en el presente estudio, se encontró que dos tercios de la población se encontraban sin cobertura de seguro subsidiado por el Estado.

Al evaluar las causas de no aseguramiento de las gestantes, diferimos con Catacora ${ }^{(20)}$; ya que no encontramos que las gestantes no se aseguren por la percepción de no cobertura financiera, observándose que todas ellas solicitaron acceder al SIS. El 80.01\% realizó esta solicitud de aseguramiento por única vez; mientras que el $19.99 \%$ la realizó en dos a más oportunidades. Los resultados de este estudio presentaron coincidencias con los encontrados en un estudio sobre "Evaluación del Efecto del SIS en los Indicadores de Salud Pública" (9), donde se señala que durante los años 2006 al 2009 hubo un incremento respecto al nivel de cobertura creciente del $15.4 \%$ al $33.8 \%$ en la población nacional. Estas cifras fueron semejantes a las cifras encontradas en este estudio, lo cual comprueba que el aseguramiento del grupo de gestantes de la DIRESA Tacna llegó al 35.26\% durante el año 2014.

Asimismo, podemos inferir que el Estado, no está cumpliendo con los objetivos de la Ley $N^{\circ} 29344^{(21)}$ "Ley de Aseguramiento en Salud", donde se considera garantizar el pleno derecho de toda la población a la seguridad social del país; priorizando a los grupos vulnerables y de riesgo social. En este tenor, el MINSA como órgano rector y los Gobiernos Regionales como órganos ejecutores no están cumpliendo sus roles a cabalidad.

En el estudio de Janice Seinfeld ${ }^{(22)}$, se encontró que las causas de no afiliación de la población fueron la falta del documento de identidad por parte del solicitante y la falta de infraestructura en los servicios de salud. Estos resultados no fueron concordantes con los nuestros, pues en este estudio se encontró que la condición civil de la gestante y el conocimiento del proceso por el personal de salud "asegurador" fueron los factores influyentes para que las gestantes no se encuentren aseguradas.

Podemos entonces concluir que en Tacna durante el año 2014 se encontró que el $64.76 \%$ de gestantes no estaban aseguradas, frente al $35.26 \%$ de gestantes aseguradas. Asimismo, existe relación significativa entre el estado civil y el aseguramiento, teniendo en cuenta que de las gestantes aseguradas, el $69.65 \%$ eran convivientes. Adicionalmente, señalamos que las gestantes solteras tienen $2.2 \%$ mayor riesgo de no asegurarse respecto a las gestantes casadas. Se evidencia también que el aseguramiento de la gestante no es oportuno y se da en mayor proporción en el último trimestre de la gestación con el $52.60 \%$ de los casos de aseguradas en el tercer trimestre. Por último, es mayor el porcentaje (58.95\%) de personal de salud responsable del aseguramiento que desconoce los procesos que el personal que los conoce (41.05\%), existiendo una relación estadística significativa entre el desconocimiento de los procesos y el bajo nivel de aseguramiento público de la gestante.

Recomendamos a nuestras autoridades implementar estrategias de aseguramiento dirigido a gestantes (campañas de aseguramiento masivas, sectorizadas, cíclica, etc.), así como de un sistema de capacitación y evaluación constante para el personal de salud responsable de los procesos de aseguramiento de la población. Finalmente, es esencial el cumplimiento de las recientes normas de aseguramiento, independiente de la evaluación socio económica y categorización de pobreza del Padrón General de Hogares. 


\section{REFERENCIAS BIBLIOGRÁFICAS}

1. Ministerio de Salud del Perú. Plan Estratégico Nacional para la Reducción de la Mortalidad Materna y Perinatal 2009-2015. Lima, Perú, 2009.

2. Del Carpio L, Situación de la mortalidad materna en el Perú, 2000-2012. RPME Salud Pública. 2013; 30(3):461-4.

3. Misas JD, Una Aproximación teórica práctica para el costeo de cápita en paquetes de salud. Universidad del Rosario. Facultad de Economía, Colombia, 2013. Disponible en:http://repository.urosario.edu.co/bitstream/handle/10336 /4844/79803024-2013.pdf?sequence=3.

4. Parodi S, Evaluando los efectos del Seguro Integral de Salud (SIS) sobre la equidad en la salud materna en el contexto de barreras no económicas al acceso a los servicios. Grupo de Análisis para el Desarrollo-GRADE. Economía y Sociedad 66, CIES, diciembre 2007. Ps 30-36. Disponible en: http://old.cies.org.pe/fies/ES/bol66/04-parodi.pdf2004:

5. Laurell C, Impacto del Seguro Popular en el sistema de salud mexicano - 1a ed. - Ciudad Autónoma de Buenos Aires: CLACSO, 2013. E-Book. ISBN 978-987-1891-70-2.

6. Ferreira B, Características del control prenatal en las embarazadas adolescentes. Montevideo -Uruguay. 2006 Informe de Salud sobre la equidad Atención de Salud para Los más Pobres.

7. Becerril V, Medina R. Sistema de salud de Brasil. Salud Pública de México [en línea] 2011, 53 (Sin mes). Disponible en:<http://www.redalyc.org/articulo.oa?id=10619779008> ISSN 0036-3634.

8. Gobierno de la República de Bolivia, Ministerio de Justicia. BOLIVIA, Informe de Progreso: Un mundo apropiado para los niños 2002-2006,2007. Disponible en: https://www.unicef.org/worldfitforchildren/files/Bolivia_WFF C5_Report.pdf.

9. Seguro Integral de Salud (S.I.S.). Evaluación del efecto del SIS en los indicadores de salud pública y gasto de bolsillo periodo 2002-2009. Gerencia de operaciones, 2011. Disponible en: http://www.sis.gob.pe/Portal/publicaciones/Consolidado_Info rme_2002-2009_07_02_2011.pdf.

10. Defensoría del Pueblo, Atención de Salud para los más pobres. Informe $\mathrm{N}^{\circ} 120$. Primera Edición. Lima -Perú, mayo de 2007. Disponible en: http://www.defensoria.gob.pe/informespublicaciones.php.

11. Santos S, Factores que limitan la cobertura de gestantes afiliadas al seguro integral de salud, en el Centro de Salud Sesquicentenario Callao - Perú 2014. UCV Callao-Perú. 2005. D i s p n ible $\quad$ e $n: \quad h t$ t $p: / /$
repositorio.ucv.edu.pe/handle/UCV/6610repositorio.ucv.edu. pe/handle/UCV/6610.

12. Munares F, Estudio sobre la calidad del Control Pre Natal en EESS del primer nivel. Lima. Tesis para optar el grado académico de Magister en Salud Pública. U.N.M.S.M., Lima-Perú. 2005. D i s p o n i b I e

e $n$ : http://cybertesis.unmsm.edu.pe/bitstream/cybertesis/1771/1 /Munares_go\%281\%29

13. Navarro J, Factores de Riesgo para el inicio tardío en la atención pre natal en gestantes que acuden al consultorio de obstetricia del Centro de Salud Leonor Saavedra. Enero a marzo 2013 . UNMSM. Lima, 2013 . Disponible en: http://cybertesis.unmsm.edu.pe/handle/cybertesis

14. Chaw I, Aseguramiento Universal Perú año 2012. Lima Perú, 2013. Disponible en: htto://slideplayer.es/slide/1106292.

15. Montalvo P, Galdos O, El efecto del Seguro Integral de Salud, sobre los Indicadores Materno Perinatales en Tacna, en los años 2002 al 2004. 2005.

16. Instituto Nacional de Estadística e Informática, Estado de la Población Peruana. Perú, 2014.

17. Vargas I, Barreras en el Acceso a la atención en salud en modelos de competencia gestionada: Colombia, 2009. D i s p o n i b I e e $n$ : https://books.google.com.pe/books?id=trzDgkuTp4sC\&pg=PA $253 \& / p g=P A 253 \& d q=$ barreras+de+acceso+a+la+atención+ingrid+vargas+lorenzo\&source $=b$

18. Instituto Nacional de Colombia, Factores que afectan el acceso y la utilización de servicios de atención prenatal estudio cualitativo en veinte departamentos. Colombia 2011-.2012. D i s p o n i b I e e $n$ : http://sinergiasong.org/cajasdeherramientas/prenatal/vinculo s/ref9-Barreras-de-Acceso.pdf

19. Saavedra B. ¿El aseguramiento público en salud, mejora la cobertura de los servicios? México,2012. Disponible en: http://www.scielo.org.mx/scielo.php?script=sci_ arttext\&pid=S0036-36342016000500561.

20. Catacora I, Estudio de Servicio de salud materna en el hospital General de Jaen, brindado a través del Seguro Integral de Salud, análisis desde el enfoque intercultural año 2012. Tomadode:http://tesis.pucp.edu.pe/repositorio/bitstream/ha ndle/123456789/5907/CATACORA_ISABEL_PASTOR_SONIA_ES TUDIO_JAEN.pdf?sequence $=1$

21. Ley $N^{\circ} 29344$, Ley de Aseguramiento Universal, publicado en el Diario El Peruano el 06 de noviembre del 2014. Disponible en: http://diariooficial.elperuano.pe/

22. Seinfeld J, Fortalecimiento del Rol Rector de la SUNASA. 2014

\section{Correspondencia}

Patricia Verónica Montalvo Chávez

E-mail: pveronicam@gmail.com

Fecha de recepción: 11 de setiembre de 2018

Fecha de aceptación: 26 de febrero de 2019 